\title{
Efeitos da associação estro-androgênica em mulheres na pós-menopausa
}

\author{
Effects of the association of estrogen and androgen in postmenopausal women
}

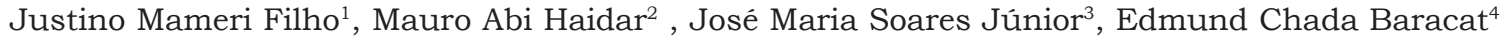

\section{RESUMO}

Objetivo: avaliar os efeitos da associação de estrogênios e androgênios sobre a qualidade de vida e a sexualidade de mulheres durante o climatério. Métodos: foram incluídas 96 pacientes com sintomas vasomotores e disfunção sexual na pós-menopausa. As mulheres foram aleatoriamente divididas em três grupos de tratamento com 32 pacientes cada: placebo, estrogênios conjugados eqüinos $(0,625 \mathrm{mg}$ por dia) e associação de estrogênios conjugados eqüinos ( $0,625 \mathrm{mg}$ por dia) e de metiltestosterona (2,5 mg por dia). O tratamento foi realizado por três meses. A avaliação da qualidade de vida e da sexualidade foi realizada por entrevista usando o Questionário de Saúde da Mulher (QSM) e o Questionário Simplificado sobre Sexualidade, antes e após o tratamento. Alguns parâmetros do risco cardiovascular, o eco endometrial e a toxidade hepática foram avaliados. Para análise dos dados aplicou-se o teste ANOVA, seguido pelo teste post hoc de Fisher e teste de Shapiro-Wilk. Resultados: houve melhora nos parâmetros do QSM nos grupos que receberam estrogenioterapia isolada e associada ao androgênio em comparação ao grupo placebo. Entretanto, não houve diferença entre os três grupos em relação às questões referentes aos sintomas somáticos. A associação estro-androgênica foi superior ao estrogênio isolado nas questões relacionadas com função sexual (média da pontuação: 64 vs 67; $\mathrm{p}<0,05$ ) e humor deprimido (média da pontuação: $75 v s$ 80; $\mathrm{p}<0,05$ ). No grupo que recebeu a terapia estro-androgênica, houve redução do colesterol total ( $212 \pm 42$ e 194 \pm 43 , antes e após o tratamento, respectivamente) e do HDL-colesterol (56 \pm 16 e 48 \pm 14 , antes e após o tratamento, respectivamente), bem como discreto aumento do eco endometrial $(4,7 \pm 2,3$ e 5,5 2,3 antes e após o tratamento, respectivamente). Não foi constatada alteração significante das enzimas hepáticas entre os grupos, durante o período de estudo. Conclusões: a associação estro-androgênica resultou em melhora na qualidade de vida e nos distúrbios sexuais. Este efeito foi superior à estrogenioterapia isolada e ao placebo. O efeito do tratamento estroandrogênico foi mais marcante nos itens de humor deprimido e de função sexual no questionário QSM.

PALAVRAS-CHAVE: Menopausa; Terapia hormonal; Sexualidade

\section{ABSTRACT}

Purpose: to evaluate the effects of the association of estrogen and androgen on the quality of life and sexuality of women during climacterium. Methods: ninety-six postmenopausal women with vasomotor symptoms and sexual dysfunction were included. The participants were randomly divided into three treatment groups with 32 pacients each: placebo, conjugated equine estrogens (CEE) ( $0.625 \mathrm{mg}$ per day) and CEE ( $0.625 \mathrm{mg}$ per day) associated with methyltestosterone ( $2.5 \mathrm{mg}$ per day). The length of the treatment period was three months. The Women Health Questionnaire (WHQ) and the Modified Sexuality Questionnaire were applied to evaluate the quality of life and sexuality before and after the treatment. Some parameters of cardiovascular risk, endometrial echo and hepatic toxicity were evaluated. ANOVA was used for data analysis followed by the Fisher test and the Shapiro-Wilk post hoc test. Results: the improvement in WHQ parameters was significant in the hormonal treatment groups (CEE and CEE + methyltestosterone) compared to the placebo group. However, there were no differences in somatic symptoms among the three groups. The association of estrogen with androgen significantly improved sexual function (score (mean): 64 vs 67, $\mathrm{p}<0.05$ ) and depressive humor (score (mean): 75 vs 80, $\mathrm{p}<0.05$ ) compared to estrogen alone. This therapy also presented a large number of WHQ questions with a high score $(\mathrm{p}<0.05)$. The use of CEE associated with methyltestosterone decreased the total cholesterol ( $212 \pm 42$ and $194 \pm 43$, before and after the treatment, respectively) and HDL colesterol ( $56 \pm 16$ and $48 \pm 14$, before and after the treatment, respectively), and slightly increased the endometrial echo ( $4.7 \pm 2.3$ and $5.5 \pm 2.3$, before and after the treatment, respectively). No signifcant changes in liver enzymes during the treatment period was detected. Conclusions: estrogen associated with methyltestosterone resulted in significant improvement in the quality of life and sexuality of postmenopausal women. This effect was superior to estrogen alone and placebo. The

\footnotetext{
Departamento de Ginecologia da Universidade Federal de São Paulo - UNIFESP (SP) e Universidade Federal do Espírito Santo - UFES (ES)

1 Professor Adjunto do Departamento de Ginecologia e Obstetrícia da Universidade Federal do Espírito Santo - UFES (ES)

2 Professor Adjunto do Departamento de Ginecologia da Universidade Federal de São Paulo - UNIFESP (SP)

3 Professor Afiliado do Departamento de Ginecologia da Universidade Federal de São Paulo - UNIFESP (SP)

4 Professor Titular do Departamento de Ginecologia da Universidade Federal de São Paulo - UNIFESP (SP)

Correspondência: José Maria Soares Júnior

Avenida Sena Madureira, 1245 apto. 11 - 04021-051 - São Paulo - SP - Telefone (Fax): (11) 5081-3685 - e-mail: jsoares415@aol.com

Recebido em: 5/11/2004 Aceito com modificações em: 29/3/2005
} 
effect of treatment with the estrogen-androgen association was evident regarding depressive humor and sexual function questions of the WHQ.

KEYWORDS: Menopause; Hormonal therapy; Sexuality

\section{Introdução}

A população feminina em todo o mundo, principalmente nos países mais desenvolvidos, vem apresentando significativo aumento da expectativa de vida. Embora a idade da menarca venha diminuindo, a da menopausa parece se manter constante ao redor dos $50 \operatorname{anos}^{1}$. Assim sendo, as mulheres vivem hoje, em média, um terço ou mais de suas vidas após a menopausa.

É conhecida a associação entre o estilo de vida e a saúde. A prevenção do desenvolvimento dos problemas de saúde, em geral, depende do estilo de vida e, portanto, dos cuidados com a mente e o corpo. Consideram-se estilo de vida adequado os cuidados que levam a uma vida emocional e interpessoal saudáveis ${ }^{2}$. Além disso, a necessidade de modificação no estilo de vida inclui: combate ao uso do fumo e do álcool, adequação nutricional, controle de peso, prática de exercício físico e cuidado com a higiene e a saúde mental ${ }^{3}$.

Sexualidade e função sexual fazem parte do bem-estar e da saúde da mulher. Assim, os aspectos da função sexual devem ser incluídos na avaliação geral de sua saúde, devendo se oferecer orientações e tratamentos adequados sempre que necessários, independentemente de sua idade 4 . A função sexual normal envolve uma série de complexos fatores hormonais, psicológicos e socioculturais ${ }^{4}$. Os esteróides sexuais são importantes na função sexual e há crescente tendência em considerá-los como sendo importantes na sexualidade feminina ${ }^{5-7}$.

O climatério representa a fase de transição que se inicia no final da menacme e se estende até a senilidade. Pode ser definido como o intervalo de tempo que compreende a transição do período reprodutivo para o não reprodutivo, sendo considerado fase de crises pelas inúmeras transformações que nele ocorrem ${ }^{8}$. A menopausa representa a última menstruação, sendo, portanto, evento que ocorre no climatério. É o último fluxo menstrual, considerado após 12 meses de amenorréia ${ }^{8-10}$.

No menacme, o estrogênio circulante é o produzindo pelos folículos ovarianos em desenvolvimento, sendo importante para a manutenção da biologia feminina. Após a ovulação, a progesterona, outro importante hormônio, é produzido pelo corpo lúteo. Já no climatério, esses hormônios são habitualmente administrados na terapia hormonal (TH), visando evitar os sintomas vasomotores ${ }^{11}$, bem como os fenômenos atróficos genitais ${ }^{12} \mathrm{e}$ da pele $^{13}$ e também a perda de massa óssea ${ }^{14}$.

Os androgênios são hormônios produzidos na mulher em três sítios principais: ovários, glândulas supra-renais e tecido adiposo periférico. Têm importância na fisiologia da mulher por serem produtos intermediários na formação dos estrogênios, por participarem da atresia dos folículos não dominantes, na produção de secreção das glândulas sebáceas e atuarem nos pêlos ${ }^{4,5}$.

No climatério, os ovários continuam a produzir testosterona, porém, os níveis plasmáticos diminuem, pois sua principal fonte, a conversão periférica da androstenediona acha-se reduzida, justificando a razão para se pensar na necessidade da inclusão dos androgênios em TH no climatério ${ }^{15}$.

A deficiência androgênica na mulher tem sido mais aceita naquelas que foram submetidas à ooforectomia bilateral, já que os ovários representam as principais fontes de testosterona circulante. Entretanto, também as mulheres climatéricas com ovários preservados apresentam niveis circulantes de testosterona progressivamente menores, podendo ter a triade de sintomas caracterizada por diminuição da libido, falta de motivação e fadiga persistente ${ }^{16}$.

Davis ${ }^{16}$ relatou importante aumento da globulina transportadora de hormônios sexuais em mulheres na pós-menopausa sob terapia estrogênica (TE) isolada. Este aumento pode atingir até 160\%, reduzindo profundamente a biodisponibilidade da testosterona. Concluiu, então, que a TE pode levar à deficiência relativa androgênica dos ovários e da supra-renal, justificando a necessidade da associação de androgênio ao estrogênio nessa fase da vida.

Os dados da literatura mostram que a sua associação ao estrogênio oferece vantagens em relação ao metabolismo ósseo, além de aumentar a libido, a atividade física e a sensação de bemestar $^{7,16}$. Estes estudos nos motivaram a avaliar os efeitos da associação de estrogênios aos androgênios na qualidade de vida e a sexualidade de mulheres na pós-menopausa.

\section{Pacientes e Métodos}

O grupo de estudo foi constituído pelas mulheres na pós-menopausa assistidas no Ambulatório de Climatério do Hospital Cassiano Antonio 
Moraes (HUCAM) da Universidade Federal do Espírito Santo (UFES), no periodo compreendido entre setembro de 1999 e dezembro de 2000.

O diagnóstico de menopausa foi baseado nos dados clínicos, ou seja, amenorréia de pelo menos 12 meses, confirmado pela elevação da gonadotrofina folículo-estimulante (FSH acima de $40 \mathrm{mUI} / \mathrm{mL}$ ). Foram incluídas pacientes com sintomas vasomotores moderados ou acentuados ou indice menopausal de Kupperman maior que 39 pontos e com queixas de disfunção sexual. Já as mulheres com contra-indicação absoluta para reposição hormonal, indices de massa corporal (IMC) maior que 30 (obesas) ou de Ferriman, Gallwey e Lorenzo maior que oito (hirsutas) foram excluídas. Além disso, as pacientes que não estavam em tratamento hormonal prévio e não fizeram uso de herbais ou dieta rica em alimentos à base de soja (uso prévio por seis meses) foram selecionadas para entrar neste estudo.

Incluímos 96 pacientes em estudo duplocego, randomizado, com duração de três meses de tratamento. Todas as pacientes foram orientadas sobre possiveis efeitos colaterais e concordaram em participar deste estudo por meio de consentimento escrito em documento formulado e aprovado pela Comissão de Ética do Centro Biomédico da Universidade Federal do Espírito Santo.

As pacientes foram, aleatoriamente, divididas em três grupos de 32 pacientes cada: GCtr (controle) recebeu placebo; GECE recebeu estrogênios conjugados eqüinos $(0,625 \mathrm{mg}$ ao dia); GEM recebeu estrogênios conjugados eqüinos $(0,625 \mathrm{mg} /$ dia $)$ associados a metiltestosterona $(2,5 \mathrm{mg} /$ dia). A doação dos medicamentos e placebo utilizados na pesquisa foi feita pela Farmácia de Manipulação Imafar-Vitória/ES. Os comprimidos tinham o mesmo aspecto, que não permitisse distinguir o conteúdo. A randomização das pacientes foi realizada com o auxílio de um programa de computador. O método utilizado para a coleta dos dados foi a entrevista.

Os sintomas foram avaliados usando-se o indice menopausal de Kupperman antes do tratamento. As pacientes foram questionadas verbalmente durante as consultas sobre os itens que compõem este indice, como fogachos, insônia, mialgia ou artralgia, formigamento, palpitação, parestesia, nervosismo, tontura, cefaléia e melancolia. Para a pontuação dos fogachos, determinamos que o sintoma leve teria freqüência menor que 5 por dia, moderado entre $5 \mathrm{e}$ 10 por dia, ao passo que os casos acentuados, mais que 10 fogachos em um dia. Os outros sintomas obedeceram aos critérios descritos por Kupperman. Foi analisado o somatório de todos os sintomas do indice menopausal para fins de avaliação da gravidade para inclusão da paciente no estudo.

Nosso protocolo de pesquisa seguiu os seguintes passos: todas as pacientes, ao se submeterem ao estudo, foram submetidas a exames de rotina para detecção precoce de câncer ginecológico (citologia cervicovaginal oncológica e mamografia), cujos resultados não serão discutidos neste trabalho. Após o exame fisico, que foi efetuado em intervalos de 4 semanas, registraram-se os valores do peso e da altura e calculou-se o IMC antes e após o tratamento. Além disto, foram pesquisadas informações sobre a idade, o tempo pós-menopausa, a raça, o hábito de tabagismo e a freqüência de doenças crônicas como o diabete melito e a hipertensão (Tabela 1).

Tabela 1 - Informações epidemiológicas e clínicas das participantes.

\begin{tabular}{|c|c|c|c|}
\hline & $\begin{array}{c}\text { GCrt } \\
\text { (placebo) }\end{array}$ & $\begin{array}{c}\text { GECE } \\
\text { (estrogênio) }\end{array}$ & $\begin{array}{c}\text { GEM } \\
\text { (estrogênio + } \\
\text { androgênio) }\end{array}$ \\
\hline Idade & $50,0 \pm 4,2$ & $51,7 \pm 3,8$ & $50,3 \pm 3,7$ \\
\hline \multicolumn{4}{|l|}{ Tempo de } \\
\hline pós-menopausa (anos) & $2,1 \pm 1,8$ & $3,0 \pm 2,3$ & $2,8 \pm 2,1$ \\
\hline \multicolumn{4}{|l|}{ Etnia $(\%)$} \\
\hline Caucasianas & 52 & 56,2 & 54,3 \\
\hline Afrobrasileiras & 8,4 & 9,6 & 7,4 \\
\hline Pardas & 39,6 & 34,2 & 38,3 \\
\hline Tabagismo (\%) & 23,7 & 28,9 & 26,3 \\
\hline Diabete (\%) & 13,2 & 15,8 & 15,8 \\
\hline Hipertensão (\%) & 15,8 & 10,8 & 13,1 \\
\hline IMC & $26,6 \pm 2,2$ & $26,1 \pm 1,7$ & $25,4 \pm 1,8$ \\
\hline
\end{tabular}

As pacientes foram submetidas à coleta de sangue para dosagens bioquímicas de glicemia de jejum, triglicérides, colesterol total e frações, enzimas hepáticas (AST, ALT e gama-GT) e FSH. Além disso, foram submetidas à avaliação ultrasonográfica, no início e no final deste trabalho. Neste estudo, o perfil lipídico foi composto pela dosagem do colesterol total e frações e dos triglicérides.

O principal instrumento da pesquisa foi o Questionário de Saúde da Mulher (QSM). É composto de 36 questões: acordo de madrugada e aí durmo mal o resto da noite (1); fico com muito medo ou apavorada sem nenhum motivo aparente (2); sintome triste e infeliz (3); fico angustiada quando saio de casa sozinha (4); perdi o interesse pelas coisas (5); sinto batedeiras (palpitações) ou a sensação de frio na barriga ou no peito (6); ainda gosto das coisas das quais gostava antes (7); acho que a vida não vale a pena (8); sinto-me nervosa ou agitada (9); tenho bom apetite (10); sinto-me inquieta e não consigo ficar parada (11); estou mais irritada que o normal (12); ficar velha me preocupa (13); sinto dores de cabeça (14); sinto-me mais cansada que o normal 
(15); tenho tonturas (16); meus seios estão doloridos ou me incomodam (17); sinto dor nas costas ou nos braços e pernas (18); tenho ondas de calor (19); estou mais atrapalhada (desastrada) que o normal (20); sinto-me bastante animada e excitada (21); tenho desconforto ou cólicas na barriga (22); sinto-me enjoada ou com vontade de vomitar (23); perdi o interesse pela vida sexual (24); tenho sensação de bemestar (25); sangro muito nas minhas menstruações (26); tenho suores a noite (27); sinto o estômago inchado (empachado) (28); tenho dificuldades para pegar no sono (29); sinto formigamento e agulhadas nos pés e nas mãos (30); estou satisfeita com a minha vida sexual (favor não responder se não tiver relação) (31); sinto-me fisicamente atraente (32); tenho dificuldades de concentração (33); minhas relações sexuais incomodam, porque minha vagina está seca (34); tenho que urinar mais vezes que o normal (35); minha memória está ruim (36). Devese salientar que os critérios de pontuação foram conforme as respostas: (1) Não, de jeito nenhum; (2) Raramente; (3) Sim, as vezes; (4) Sim, sem dúvida. Há 30 questões referentes a algo desfavorável, como por exemplo: sentir-se triste ou infeliz, e seis questões $(7,10,21,25,31$ e 32$)$ que se referem a algo favorável, como estar satisfeita com sua vida sexual. As questões do QSM são divididas em nove dimensões: função sexual (questões: 24, 31, 34), atração $(13,21$, $32)$, distúrbios do sono $(1,11,21)$, sintomas vasomotores $(19,27)$, dificuldades cognitivas $(20,33$, $36)$, sintomas menstruais $(17,22,26,28)$, sintomas somáticos $(14,15,16,18,23,30,35)$, humor deprimido $(3,5,7,8,10,12,25)$ e ansiedade $(2,4,6,9)$. Neste estudo, foram avaliadas 32 questões do QSM, já que as quatro questões referentes aos sintomas menstruais não foram aplicadas, em função de as mulheres incluídas estarem na pós-menopausa.

As questões foram respondidas antes do início e após três meses de tratamento. Nos itens desfavoráveis do QSM, quando houve melhora, a pontuação foi menor. Caso contrário, o aumento dos pontos indica piora na qualidade de vida em relação àqueles itens. Já quanto aos itens favoráveis, a melhora é identificada pela elevação da pontuação.

$\mathrm{Na}$ avaliação por grupos de sintomas (dimensões do QSM), e também na avaliação global do QSM, para uniformizar os dados, utilizou-se o recurso de inverter a pontuação das questões favoráveis, para que todas as questões tivessem pontuação inicial maior e que diminuíssem com a melhora na qualidade de vida. Desta forma, quanto menor a pontuação, tanto melhor será a qualidade de vida.

Também foi utilizado o Questionário Simplificado sobre Sexualidade (McCoy modificado), baseado no questionário utilizado por De Paula et al. ${ }^{17}$. Este questionário analisa questões referentes à freqüência de relações sexuais, libido, orgasmo, dispareunia e sangramento no ato sexual. A questão referente à freqüência de relações sexuais tem a seguinte pontuação: 1 - menos que uma vez por mês; 2 - uma vez por mês; 3 - uma vez de quinze em quinze dias; 4 uma vez por semana; 5 - duas vezes por semana; 6 três vezes por semana; Já as outras questões são pontuadas da seguinte forma: 1 - não; 2 - às vezes; 3 - sim; Esse questionário foi aplicado antes e após três meses de tratamento. Quando houver melhora dos parâmetros, haverá aumento na pontuação.

A comparação das pontuações antes e após o tratamento foi feita pela análise de variância (ANOVA) de uma via para o modelo completamente randomizado. No caso de existir diferença significante entre os grupos, aplicou-se o teste post hoc de Fisher ( $t$ protegido) para a comparação intergrupos. Antes da realização da análise de variância da ANOVA, aplicou-se o teste de Shapiro-Wilk para verificação da normalidade da distribuição dos dados. Aceitou-se a hipótese de distribuição normal dos dados e de variâncias iguais quando os valores foram superiores a $p>0,10$. Em todas as situações a hipótese de igualdade de variâncias e ajuste pela curva normal foi aceita. Toda a análise estatística dos dados foi feita com o programa GB Stat versão 6.5 (Dynamic Microsystems, Inc., USA, 1997). Em todos os testes de comparação, fixou-se o nivel de rejeição da hipótese de nulidade em 0,05 ou $5 \%$.

\section{Resultados}

Na avaliação de cada questão do QSM isoladamente, antes e após os tratamentos, observou-se diferença significativa na pontuação dos grupos GCtr, GECE e GEM. Os resultados do GEM foram melhores do que os do GCtr em relação às questões referentes a função sexual, atração, distúrbio do sono, sintomas vasomotores, dificuldades cognitivas, humor deprimido e ansiedade. Apenas quanto às questões referentes aos sintomas somáticos, não houve diferença significante entre os três grupos.

Já na comparação entre os grupos GECE e GCtr, houve melhora significante nas mulheres que receberam estrogênio em quase todas as questões, exceto nas relacionadas com os sintomas somáticos $(14,16,18$ e 23). Entre os grupos GECE e GEM, houve diferença quanto ao humor deprimido e função sexual (Tabela 2).

A avaliação do QSM em suas oito dimensões (sintomas menstruais foram desconsiderados) demonstrou redução significativa da pontuação com uso de placebo em três dimensões, e em sete dimensões com estrogênio isolado e com a associação estro-androgênica (Tabela 2). As dimensões função sexual e humor deprimido tiveram melhora significativa com a combinação estrogênio-androgênio em relação à estrogenioterapia isolada. 
Tabela 2 - Média de pontuação do questionário QSM nos três grupos, antes e após o tratamento.

$\begin{array}{ccc}\text { GCrt } & \text { GECE } & \text { GEM } \\ \text { (placebo) } & \text { (estrogênio) } & \begin{array}{c}\text { (estrogênio + } \\ \text { androgênio) }\end{array}\end{array}$

\begin{tabular}{lrrrrrl} 
Dimensões & Antes & Após & Antes & Após & Antes & Após \\
\hline Função sexual & 88,7 & 70,7 & 95,7 & 66,7 & 111,7 & $64,0^{*, \#}$ \\
Atração & 100,3 & 92,7 & 113,0 & $78,3^{*}$ & 115,7 & 81,7 \\
Distúrbios do sono & 104,0 & 88,3 & 116,0 & $74,3^{*}$ & 119,7 & $62,0^{*}$ \\
Sintomas vasomotores & 110,5 & 72,0 & 130,0 & $49,5^{*}$ & 124,5 & $51,5^{*}$ \\
Dificuldades cognitivas & 97,3 & 92,7 & 105,7 & $80,7^{*}$ & 104,0 & $75,0^{*}$ \\
Sintomas somáticos & 98,9 & $81,7^{*}$ & 101,3 & $75,4^{*}$ & 102,9 & $72,9^{*}$ \\
Humor deprimido & 107,4 & $90,3^{*}$ & 117,3 & $79,7^{*}$ & 123,9 & $75,0^{*}, \#$ \\
Ansiedade & 98,8 & $82,3^{*}$ & 110,0 & $68,5^{*}$ & 111,8 & $62,8^{*}$ \\
\hline *p< 0,05 (significante, comparando antes e após tratamento). & & \\
\#p < 0,05 (significante em relação aos grupos placebo, estrogênio e estrogênio + \\
androgênio). \\
GCtr = grupo controle (placebo). \\
GECE = estrogênios conjugados eqüinos (0,625 mg ao dia). \\
GEM estrogênios conjugados eqüinos (0,625 mg/dia) associado a metiltestosterona \\
(2,5 mg/dia).
\end{tabular}

Na avaliação global do QSM, observa-se que não houve diferença entre os grupos antes do tratamento, demonstrando que a qualidade de vida dos três grupos era semelhante. Após o período de tratamento, notou-se queda da pontuação em todos os grupos (GCtr: 99,1 $\pm 19,9$ para 82,6 $\pm 18,9$; GECE: $107,0 \pm 23,5$ para $72,6 \pm 18,4$; GEM: $110,0 \pm 22,3$ para $69,7 \pm 18,6)$. Estes dados sugerem que o placebo também determinou melhora na qualidade de vida. Contudo, as pontuações dos questionários respondidos pelas mulheres do grupo GECE e do GEM foram significativamente menores do que no GCtr.

Como a resposta ao questionário é subjetiva, decidiu-se separar todas as pacientes em apenas duas categorias: as que apresentaram melhora efetiva e as que não apresentaram melhora efetiva ao longo do tratamento, em cada questão. Con- siderou-se como melhora efetiva uma redução de, pelos menos, dois pontos em determinada questão. Assim, cada paciente apresentou ao final do tratamento determinado número de questões nas quais se identificou melhora efetiva, e outro número de questões sem alteração ou piora.

Observou-se que no GCtr ocorreu melhora em $7,4 \pm 4,8$ questões. O número de questões com melhora foi significativamente maior nos grupos $\operatorname{GECE}(11,6 \pm 4,8)$ e GEM $(14,5 \pm 6,4)$. A análise estatística mostrou melhora com ambos os tratamentos em relação ao placebo; na comparação entre os grupos, a associação estro-androgênica mostrou-se significativamente melhor $(\mathrm{p}<0,05)$ que a estrogenioterapia isolada.

A análise do Questionário sobre Sexualidade é feita na Tabela 3, com a associação estroandrogênica demonstrando melhora significativa em relação ao placebo em todos as cinco questões, e em relação à estrogenioterapia isolada nas questões libido e orgasmo.

Tabela 3 - Pontuação do questionário sobre sexualidade nos três grupos, antes e após 0 tratamento.

$$
\begin{array}{ccc}
\text { GCrt } & \text { GECE } & \text { GEM } \\
\text { (placebo) } & \text { (estrogênio) } & \text { (estrogênio + }
\end{array}
$$
androgênio)

\begin{tabular}{lrrrrrr} 
Questões & Antes & \multicolumn{1}{c}{ Após } & Antes & Após & Antes & Após \\
\hline Frequiência sexual & 143 & 151 & 112 & 126 & 133 & $166^{*}$ \\
Libido & 53 & 59 & 49 & 56 & 54 & $81^{*}$,\# \\
Orgasmo & 62 & 70 & 57 & 66 & 56 & $81^{*}$,\# \\
Dispareunia & 38 & 48 & 34 & 43 & 36 & $61^{*}$ \\
Sangramento ao coito & 31 & 33 & 28 & 32 & 34 & $42^{*}$
\end{tabular}

*Significante em relação ao placebo.

*\# Significante em relação ao placebo e ao estrogênio.

$\mathrm{GCtr}=$ grupo controle (placebo)

GECE = estrogênios conjugados eqüinos $(0,625 \mathrm{mg}$ ao dia).

GEM estrogênios conjugados eqüinos $(0,625 \mathrm{mg} / \mathrm{dia})$ associados a metiltestosterona (2,5 mg/dia).

Tabela 4 - Glicemia de jejum, perfil lipídico, toxicidade hepática e eco endometrial antes e após o tratamento.

\begin{tabular}{lcccccc}
\hline & \multicolumn{2}{c}{$\begin{array}{c}\text { GCrt } \\
\text { (placebo) }\end{array}$} & \multicolumn{2}{c}{$\begin{array}{c}\text { GECE } \\
\text { (estrogênio) }\end{array}$} & \multicolumn{2}{c}{$\begin{array}{c}\text { GEM } \\
\text { (estrogênio + androgênio) }\end{array}$} \\
& Antes & Após & Antes & Após & Antes & Após \\
\hline Glicemia (mg/dL) & $107,7 \pm 64,3$ & $101,6 \pm 37,2$ & $99,3 \pm 16,4$ & $97,5 \pm 16,3$ & $102,8 \pm 31,2$ & $100,7 \pm 35,3$ \\
Colesterol total (mg/dL) & $215,5 \pm 41,4$ & $212,4 \pm 31,6$ & $205,1 \pm 49,3$ & $198,5 \pm 36,8$ & $211,7 \pm 42,4$ & $194,2 \pm 42,9^{*}$ \\
HDL colesterol (mg/dL) & $59,3 \pm 12,6$ & $56,0 \pm 10,6$ & $55,9 \pm 14,9$ & $56,7 \pm 15,3$ & $56,1 \pm 15,8$ & $48,3 \pm 13,5^{*}$ \\
LDL colesterol (mg/dL) & $132,1 \pm 34,9$ & $127,4 \pm 27,9$ & $118,7 \pm 36,2$ & $115,7 \pm 34,8$ & $133,8 \pm 30,5$ & $123,0 \pm 31,8^{*}$ \\
Triglicerídeos (mg/dL) & $131,6 \pm 62,9$ & $138,7 \pm 64,3$ & $141,9 \pm 79,0$ & $154,6 \pm 87,3$ & $118,7 \pm 61,9$ & $119,5 \pm 63,7$ \\
TGO (mU/mL) & $18,2 \pm 3,5$ & $19,6 \pm 3,9$ & $21,9 \pm 4,2$ & $20,1 \pm 4,7$ & $19,6 \pm 4,3$ & $18,8 \pm 4,9$ \\
TGP (mU/mL) & $23,1 \pm 5,2$ & $22,7 \pm 4,8$ & $25,3 \pm 5,7$ & $27,1 \pm 4,9$ & $22,9 \pm 5,5$ & $24,1 \pm 5,9$ \\
Gama GT (Ul/mL) & $15,0 \pm 4,4$ & $17,3 \pm 5,1$ & $20,3 \pm 5,1$ & $18,6 \pm 4,7$ & $17,9 \pm 5,2$ & $18,3 \pm 5,7$ \\
Endométrio (mm) & $5,7 \pm 4,0$ & $5,3 \pm 3,1$ & $5,2 \pm 3,1$ & $7,6 \pm 4,7^{*}$ & $4,7 \pm 2,3$ & $5,5 \pm 2,3^{*}$
\end{tabular}

${ }^{*} \mathrm{p}<0,05$ (quando comparado os dados antes e após o tratamento).

$\mathrm{GCtr}=$ grupo controle (placebo).

GECE = estrogênios conjugados eqüinos $(0,625 \mathrm{mg}$ ao dia).

GEM = estrogênios conjugados eqüinos $(0,625 \mathrm{mg} / \mathrm{dia})$ associados a metiltestosterona $(2,5 \mathrm{mg} / \mathrm{dia})$. 
Na Tabela 4 observam-se os valores da glicemia de jejum, do perfil lipídico, das enzimas hepáticas e do eco endometrial. Não foi constatada diferença significante na dosagem de glicemia, triglicerídeos, AST, ALT e gama-GT, quando comparados os valores antes e após o tratamento nos três grupos estudados. Contudo, houve diminuição significante dos niveis de colesterol total e das frações HDL e LDL após o tratamento no grupo que recebeu androgênio. Além disso, foi identificado aumento significante do eco endometrial nas mulheres dos grupos GECE e GEM.

\section{Discussão}

A maior eficácia da associação estrogênioandrogênio quando comparada à estrogenioterapia isolada na TH do climatério já foi mostrada em vários estudos ${ }^{7,11,17,18}$, especialmente em relação à prevenção da osteoporose e melhora da atividade física, da sensação de bem-estar, do humor, da depressão e da função sexual, resultando em melhora da qualidade de vida ${ }^{7,11,16,17}$.

Os androgênios têm efeito anabolizante importante, melhorando a força muscular e o dinamismo das pacientes ${ }^{18}$. A adinamia é fator que afeta negativamente a qualidade de vida, sobretudo na pós-menopausa. As mulheres que receberam reposição androgênica percebem importante mudança no nivel de energia e disposição para o trabalho, melhorando também a libido ${ }^{7,16-18}$.

Em nosso estudo, na avaliação individual do QSM, observamos melhora significante das queixas com a associação estro-androgênica em 22 questões e com a estrogenioterapia isolada em 14 questões em comparação ao grupo placebo. Estes resultados confirmam os de outros trabalhos ${ }^{19-24}$, mostrando melhora na qualidade de vida com a associação estrogênio-androgênio, que é superior à estrogenioterapia isolada e ao placebo, nos itens relacionados à atividade física, humor, depressão e função sexual.

A melhora dos distúrbios sexuais com a associação de androgênios aos esquemas de TH é tema de muitas controvérsias. Nos últimos anos vários autores ${ }^{7,16-18}$ têm referido que a associação de androgênios à TH no climatério em pequenas doses não apresenta efeitos colaterais importantes ou irreversiveis, sendo benéfica para as mulheres que têm distúrbios da função sexual.

Nossos resultados são concordantes com os de vários autores ${ }^{7,17-18,22}$. Podemos, pois, sugerir que o uso de $2,5 \mathrm{mg}$ de metiltestosterona associados a $0,625 \mathrm{mg}$ de estrogênios conjugados eqüinos alterou positivamente as respostas relacionadas à libido e ao orgasmo em mulheres na pós-menopausa.
Em todos os grupos houve redução na pontuação e conseqüentemente melhora na qualidade de vida. Ambos os tratamentos (estrogênio isolado e combinação estrogênio-androgênio) foram capazes de produzir melhora na qualidade de vida superior ao placebo. Observou-se redução maior da pontuação no grupo submetido à terapia com a associação estrogênio-androgênio em relação ao estrogênio isolado.

Questiona-se a segurança da associação de androgênios à TH no climatério em relação à toxicidade hepática, ao perfil lipídico, à sua ação no endométrio e se os benefícios sobre a qualidade de vida e sexualidade poderiam ser obtidos com doses fisiológicas de metiltestosterona por via oral, entre 1,25 e 2,5 mg/dia, sem efeitos androgênicos importantes. Em revisão sobre este tema ${ }^{20-23}$, pode-se concluir que doses menores ou iguais a $5 \mathrm{mg} /$ dia são seguras em relação à toxicidade hepática e aos efeitos virilizantes. Quando estes últimos efeitos aparecem, podem ser reversíveis após a interrupção do tratamento ${ }^{22-23}$. Gitlin et $\mathrm{al}^{24}$ avaliaram a função hepática periodicamente por 24 meses após o início da associação de $0,625 \mathrm{mg}$ de estrogênios conjugados e de 1,25 mg de metiltestosterona, e também de 1,25 mg de estrogênios conjugados e de 2,5 mg de metiltestosterona. Estes autores não detectaram hepatotoxicidade com estes esquemas de tratamento. Em nosso estudo, não encontramos aumento significante das enzimas hepáticas após três meses de tratamento. Não foram observados também efeitos androgênicos importantes com a reposição estro-androgênica.

Em nosso estudo, a administração de androgênios na TH causou redução significante de HDL-colesterol, como já havia sido detectado em estudo anterior ${ }^{17}$. Foi observada também, no nosso trabalho, redução do colesterol total e do LDLcolesterol nas usuárias da associação estroandrogênica. Não registramos alteração nos níveis plamáticos de glicose e triglicerídios.

Indícios de proliferação do endométrio apareceram na avaliação após seis meses de tratamento nos grupos tratados com estrogenioterapia isolada e com a associação estro-androgênica. Assim, o acompanhamento destas pacientes pela ultra-sonografia endovaginal semestralmente seria necessário. O espessamento endometrial foi menor no grupo com associação estro-androgênica do que com estrogenioterapia isolada.

Houve melhora significante na qualidade de vida com a utilização da terapia estrogênica isolada e com a associação estrogênio-androgênio, superiores ao placebo, com exceção das questões referentes aos sintomas somáticos. Entre estes dois esquemas terapêuticos, a associação estroandrogênica mostrou-se superior, especialmente 
em relação à depressão e ao humor, à atividade física e à função sexual. A utilização de baixa dose de metiltestosterona (2,5 $\mathrm{mg}$ ao dia) associada a $0,625 \mathrm{mg}$ de estrogênios conjugados eqüinos mostrou-se segura em curto prazo em relação a alguns parâmetros de risco cardiovascular e toxicidade hepática, sem efeitos colaterais graves.

\section{Referências}

1. McKinlay SM, Brambilla DJ, Posner JG. The normal menopause transition. Maturitas. 1992;14(2):103-15.

2. Halbe HW, Fonseca AM. Síndrome do climatério. In: Halbe HW, editor. Tratado de ginecologia. $2^{a}$ ed. São Paulo: Roca; 1994. p. 1243-58.

3. Carr AJ, Thompson PW, Kirwan JR. Quality of life measures. Br J Rheumatol. 1996;35(3):275-81.

4. Davis SR. The clinical use of androgens in female sexual disorders. J Sex Marital Ther. 1998;24(3):153-63.

5. Sherwin BB, Gelfand MM. The role of de androgen in the maintenance of sexual functioning in oophorectomized women. Psychosom Med. 1987;49(4):397-409.

6. Kaplan HS, Owett T. The female androgen deficiency syndrome. J Sex Marital Ther. 1993;19(1):3-24.

7. Sarrel P, Dobay B, Wiita B. Estrogen and estrogenandrogen replacement in postmenopausal women dissatisfied with estrogen-only therapy. J Reprod Med. 1998;43(10):847-56.

8. Rodrigues de Lima G, Baracat EC. Sindrome do climatério: conceito, fisiopatologia, quadro clínico e diagnóstico. In: Rodrigues de Lima G, Baracat EC, editores. Ginecologia endócrina. São Paulo: Atheneu; 1995. p. 253-98.

9. Baracat EC, Simões MJ, Soares JM Jr, Haidar MA, Rodrigues de Lima G. Ultrastructural aspects of the postmenopausal endometrium after oral or transdermal estrogen administration. Clin Exp Obstet Gynecol. 2001;28(1):26-30.

10.Haidar MA, Baracat EC, Simões MJ, Focchi GR, Evencio Neto J, de Lima GR. Premature ovarian failure: morphological and ultrastructural aspects. Sao Paulo Med J. 1994;112(2):534-8.

11.Hammond CB. Menopause and hormone replacement therapy: an overview. Obstet Gynecol. 1996;87(2 Suppl):2S-15S.
12. Girão MJBC, Sartori MGF. Alterações do trato urinário inferior. In: Rodrigues de Lima G, Baracat EC, editores. Ginecologia endócrina. São Paulo: Atheneu; 1995. p. 271-2.

13. Silva de Sá MF. Repercussões do climatério sobre a pele e o colágeno. In: Marinho RM, editor. Climatério. Rio de Janeiro: Medsi; 1995. p. 39-41.

14.Lindsay R. The menopause and osteoporosis. Obstet Gynecol. 1996;87(2 Suppl):16S-19S.

15.Zumoff B, Strain GW, Miller LK, Rosner W. Twentyfour-hour mean plasma testosterone concentration declines with age in normal premenopausal women. J Clin Endrocrinol Metab. 1995;80(4):1429-30.

16.Davis SR. The therapeutic use of androgens in women. J Steroid Biochem Mol Biol. 1999;69(1-6):177-84.

17.De Paula PJF, Haidar MA, Nunes MG, Soares Junior JM, Baracat EC. Effects of methyltestosterone on lipid profile in postmenopausal patients. Climacteric. 2002;5 Suppl 1:167.

18.Yialamas MA, Hayes FJ. Androgens and the ageing male and female. Best Pract Res Clin Endocrinol Metab. 2003;17(2):223-36.

19.Dobs AS, Nguyen T, Pace C, Roberts CP. Differential effects of oral estrogen versus oral estrogenandrogen replacement therapy on body composition in postmenopausal women. J Clin Endocrinol Metab. 2002;87(4):1509-16.

20.Adamson DL, Webb CM, Collins P. Esterified estrogens combined with methyltestosterone improve emotional well-being in postmenopausal women with chest pain and normal coronary angiograms. Menopause. 2001;8(4):233-8.

21.Barrett-Connor E, Young R, Notelovitz M, Sullivan J, Wiita B, Yang HM, et al. A two-year, double-blind comparison of estrogen-androgen and conjugated estrogens in surgically menopausal women. Effects on bone mineral density, symptoms and lipid profiles. J Reprod Med. 1999;44(12):1012-20.

22.Gelfand MM, Wiita B. Androgen and estrogen-androgen hormone replacement therapy: a review of the safety literature, 1941 to 1996. Clin Ther. 1997;19(3):383-404.

23.Slayden SM. Risks of menopausal androgen supplementation. Semin Reprod Endocrinol. 1998;16(2):145-52.

24.Gitlin N, Korner P, Yang HM. Liver function in postmenopausal women on estrogen-androgen hormone replacement therapy: a meta-analysis of eight clinical trials. Menopause. 1999;6(3):216-24. 